\title{
Dissecting graphene capacitance in electrochemical cell
}

\author{
Sheng Sun ${ }^{\mathrm{a}, \mathrm{b}}$, Yue $\mathrm{Qi}^{\mathrm{c}}$, Tong-Yi Zhang ${ }^{\mathrm{a}, *}$ \\ ${ }^{a}$ Department of Mechanical and Aerospace Engineering, Hong Kong University of Science and Technology, \\ China SAR \\ ${ }^{b}$ Department of Engineering Mechanics, College of Mechanics and Materials, Hohai University, Nanjing \\ 210098, China \\ ${ }^{c}$ Chemical Engineering and Materials Science Department, Michigan State University, East Lansing, \\ 48824,USA, yueqi@egr.msu.edu
}

\begin{abstract}
Quantum capacitance of graphene plays a significant role for graphene's applications in electrochemical devices and sensors, while the determination of these basic characters of Dirac point, Fermi energy, quantum capacitance, etc is still a subject of considerable debate in both experiments and simulations. Here, we report joint firstprinciples/continuum calculations (JFPCCs) on a monolayer graphene electrode immersed in an electrolyte coupled with a reference electrode under an applied potential. The JFPCCs gave the Fermi level, charge density on graphene, Dirac point energy, electrostatic potential, electric double layer etc as a function of the applied potential with respect to the reference electrode. These results revealed the strongly coupled relationship between Fermi level change and Dirac point shift in electrochemical cell. The total capacitance of the electrochemical cell was dissected into the quantum capacitance of the graphene electrode and the capacitance of the electric double layer. Furthermore, simple and analytic formulas were proposed for the three capacitances, which predicted, in sufficient accuracy, the behavior of capacitance versus potential. These findings deepen the understanding of quantum capacitance of graphene, which will stimulate novel experimental and theoretical studies and boost the applications of graphene in electrochemical and energy areas.
\end{abstract}

Keywords: Graphene, Quantum capacitance, Dirac point shift, Electrode/electrolyte

*Corresponding author
Email address: mezhangt@ust.hk. Tel: $\quad+852 \quad 2358-7192$. (Tong-Yi Zhang)

Preprint submitted to Electrochimica Acta

February 6, 2015 
interface, Joint first-principles/continuum calculations

\section{Introduction}

With a single layer of hexagonal $s p^{2}$ hybridized carbon network, pristine graphene behaves as a zero-gap semiconductor with conically shaped valence and conduction band tips joined at the Dirac point [1, 2]. Due to its extremely high surface area and high conductivity, graphene became an ideal choice as electrodes in super capacitors for portable energy storage devices [3]. The total capacitance of a graphene electrode in an electronic device or an electrochemical cell is upper-bounded by an intrinsic capacitance called the quantum capacitance, $C_{Q}$, due to the limited density of states (DOS) or limited charge carrier density near the Dirac point [4, 5]. The quantum capacitance was formerly considered as the contribution of electrode to the capacitance of the Helmholtz layer at the electrode/electrolyte interface due to electronic response in electrode [6, 7]. The application of graphene in the next generation electronic devices, including super capacitors, requires deliberate control of the density of the charge carriers, Fermi energy, and Dirac point [8, 9]. For example, the Dirac point and the density of charge carriers of graphene can be tuned by introducing dopants through solution processing or adsorption [10, 11]. On the other hand, utilizing the Dirac point shift, graphene can also be used as biosensors [12] and pH-sensors [13, 14]. However, the determination of these basic electronic and electrochemical characters (Dirac point, Fermi energy, quantum capacitance ... ) is still a subject of considerable debate for both experiments and simulations [15]. These properties, although intrinsic to graphene, have to be measured with a proper reference and in an electrochemical environment. For example, $C_{Q}$ of graphene was experimentally determined with the field-effect transistor (FET) configuration [16, 17, 18, 19, 20, 21, 22], in which a graphene sample was placed on a substrate and connected to source and drain electrodes and the current through the graphene was controlled by a gate potential [16, 17, 18, 21, 22, 23]. In experiments, the total capacitance is measured and the quantum capapcitance is calculated by using $\frac{1}{C_{\text {total }}}=\frac{1}{C_{g}}+\frac{1}{C_{Q}}$, based on the value of geometry capacitance $C_{g} . C_{g}$ is usually treated as a parallel plate capacitor and calculated by using $C_{g}=\epsilon_{0} \epsilon / t$, where $\epsilon_{0}$ and 
$\epsilon$ are the vacuum dielectric constant and the nominal dielectric constant of the gate dielectric material, respectively, and $t$ is the thickness of the plate. In the FET configuration, however, the capacitance due to the substrate solid gate dielectric materials, such as metal-oxides, $\mathrm{Si}$, and $\mathrm{hBN}$, might play an overwhelming role in the experimental attempts to measure $C_{Q}$. More recently, solution-gated field-effect transistors (SGFET) were employed [4, 5, 10, 19, 20]. The key merit of immersing graphene in solution in SGFET measurements is that $C_{Q}$ can be dominant due to the small value of Debye screening length. In the SGFET measurements of $C_{\text {total }}$ [4, 5, 19, 20], ionic liquid was usually employed as solution and only its Gouy-Chapman capacitance was considered, which might induce large deviations in the determination of $C_{Q}$ [24, 25]. Fewer work was conducted in aqueous solution to evaluate quantum capacitance due to difficulties to evaluate capacitance at the electrode/electrolyte interface including both Gouy-Chapman layer and Stern layer.

Figure 1A schematically shows an electrochemical cell with a monolayer graphene immersed in an electrolyte coupled with a reference electrode. The electrochemical reaction is expressed by Graphite $\pm n e=$ Graphite $^{n \mp}$. The total capacitance of the electrochemical cell, $C_{t o t a l}$, is the quotient of the charge stored inside the graphene electrode over the potential across the graphene electrode and the reference electrode, as shown in Figure 1B. In this configuration, the total capacitance can be divided into two in serial capacitances, the quantum capacitance $\left(C_{Q}\right)$ of graphene and the capacitance $C_{d l}$ of the electric double layer at the graphene/electrolyte interface, and thus $\frac{1}{C_{\text {total }}}=\frac{1}{C_{d l}}+\frac{1}{C_{Q}}$. Excess negative electrons/positive holes will be generated in graphene under an applied sustained potential defined by $\mathcal{E}=\left(\bar{\mu}^{(R)}-\bar{\mu}^{(W)}\right) /|e|$, where $\bar{\mu}^{(W)}$ and $\bar{\mu}^{(R)}$ are the electron chemical potentials of the working electrode (graphene) and the reference electrode, respectively. When the electron chemical potential of the reference electrode is set to be zero, $\mathcal{E}=-\bar{\mu}^{(W)} /|e|$. In electrochemistry, the potential of zero charge, $\mathcal{E}_{\mathrm{PZC}}$, at which an electrode is charge-neutral, is widely used as a reference state. The excess negative electrons/positive holes in graphene cause a redistribution of ions in the electrolyte and shift the DOS of graphene up/down, respectively. The excess charge-induced DOS shift is expressed by the Dirac point shift, $\Delta \varepsilon$. The shift of the Dirac point changes with the electron density in the graphene and 
the charge density in the electrolyte, instead of only depending on the electron density or the Fermi energy in vacuum. This shift becomes extremely important for measuring graphene electronic properties with SGFET and for electrochemical applications of graphene such as super capacitors, biosensors [12] and pH-sensors [13, 14]. The excess charge along with the shift of the Dirac point is determined by the Fermi level of graphene with respect to the Dirac point, which is called the chemical potential of electrons in graphene, $\mu$. Thus, the electron chemical potential of the graphene electrode is given by $\bar{\mu}^{(W)}=\bar{\mu}_{\mathrm{PZC}}^{(W)}+\mu+\Delta \varepsilon$. Figures $1 \mathrm{C}$ and $1 \mathrm{D}$ further show, respectively, the negative, when $\mathcal{E}<\mathcal{E}_{\mathrm{PZC}}$, and positive, when $\mathcal{E}>\mathcal{E}_{\mathrm{PZC}}$, charge states of the graphene, where $-|e|\left(\mathcal{E}-\mathcal{E}_{\mathrm{PZC}}\right)=\mu+\Delta \varepsilon$.

Although some experimental [5, 10, 19, 20] and theoretical [26] work has been conducted to investigate the total capacitance and quantum capacitance of graphene in solution, the strongly coupled shifts of Fermi level, Dirac point, and charge density on graphene have never been illustrated clearly, especially in aqueous solution. Computing charged graphene in electrolyte under an applied potential is non-trivial. Traditionally, charged graphene is simulated with a uniform compensating charge background to keep the representative unit cell in charge-neutral state, which is obviously far from the real experimental conditions and excludes the effect of electrolyte. Using the uniform compensating charge background method, Topsakal et al. [27, 28, 29] showed that the Fermi level changed accordingly with the excess charge in graphene but the method was suffered from an artificial effect of electrons leaking into the vacuum zone [28, 29]. Recently, Wood et al. studied the effects of local structures and morphology of graphene on $C_{Q}$ by coupling first-principles calculations with the effective screening medium method [30]. However, all the results were only computed with respect to the chemical potential of electrons in graphene $\mu$ [31], instead of an applied electrode potential $\mathcal{E}$.

In this letter, an electrochemical cell with graphene immersed in electrolyte under an applied potential was properly modeled by the recently developed joint density functional theory (JDFT) [32, 33]. In JDFT, the same electrostatic potential is involved in the DFT and the modified Poisson-Boltzmann equation (See Appendix). Thus, the electron density distribution and the bound charge (or ion) distribution are simultane- 
ously obtained in JDFT. In addition, JDFT includes contributions from both Stern layer and Gouy-Chapman layer due to the localization of dielectric constant and screening. Our JDFT calculations clearly demonstrated the Dirac point shift is attributed to an electrostatic potential $\phi$ via $-|e| \phi=\Delta \varepsilon$ when the graphene thickness was appropriately defined. Based on this relationship, a simple model was proposed to unify the strongly coupled relationship among Dirac point shift, electrostatic potentials due to electrostatic double layers, Fermi energy, and both the quantum and double layer capacitances.

\section{Computations}

All calculations were performed by using the open-source software JDFTx [34] with the plane wave basis and periodic boundary conditions. In all calculations, the length along the $z$ direction (perpendicular to the graphene plane) of the primitive cell was about $40 \AA$ and the region out of the graphene was full of electrolyte. Various electrolytes were modeled with different nominal dielectric constants and ionic concentrations. Two carbon atoms were included in the unit cell with their spacing of $d$. The lattice vectors in the graphene plane had the angle of $60^{\circ}$ and the length of $\sqrt{3} d$. The revised Tao-Perdew-Staroverov-Scuseria (revTPSS) metageneralized gradient approximation (mGGA) of exchange-correlation functional [35], which was promise in DFT calculations for surface properties [36], and the norm-conserving pseudopotential with Troullier-Martins scheme [37] were employed in the present work. The MonkhorstPack $k$-point meshes [38] were of $60 \times 60 \times 1$ grids and the cutoff energy was 40 Hartree ( 1 Hartree $\approx 27.2114 \mathrm{eV}$ ). The valence electronic configuration for $\mathrm{C}$ was $2 \mathrm{~s}^{2} 2 \mathrm{p}^{2}$. The temperature was set to be $298 \mathrm{~K}$. Electron densities, ion positions and the length of lattice vectors were all allowed to relax in all calculations. The conjugate gradient scheme [39] was used to minimize the total energy of the calculated system. The approximate ground-state bands and electron densities were obtained at the equilibrium state. The relative accuracies in the energy differences were set to be $10^{-7}, 10^{-6}$ and $10^{-6}$ Hartree in two successive iterative steps for electron densities, ion positions and lattice length changes respectively. The computed equilibrium $\mathrm{C}-\mathrm{C}$ bond length of 
charge-free graphene was $1.420 \AA$, nearly the same with the experimental results [40].

In the present JDFT calculations, the excess charge on graphene was computed with an applied sustained $\bar{\mu}^{(W)}$ (or equivalently $\mathcal{E}$ ). Alternately, the $\bar{\mu}^{(W)}$ could be computed with a pre-set value of excess charge on graphene. Since the solution was under electrochemical equilibrium, both methods yielded the same results, while the latter converged faster. The total DOS of graphene with a value of excess charges was computed by using linear-tetrahedron sampling method [41]. The amount of excess electron, $q$, was also confirmed by integrating the DOS from the Dirac Point at $\varepsilon_{D}$ to the Fermi level $\bar{\mu}$.

\section{Results and discussion}

The JDFT calculations give the Fermi level $\bar{\mu}^{(W)}$, the energy level of the Dirac point $\varepsilon_{D}$ and the DOS of the graphene at equilibrium with respect to an applied potential. Figure 2A shows the computed DOS of the charge-free graphene in the electrolyte with nominal dielectric constant of 78.4 and ion concentration of $1.0 \mathrm{M}$, which yields the electrode potential of zero charge $(\mathrm{PZC}) \mathcal{E}_{\mathrm{PZC}}=-\bar{\mu}^{(W)} /|e|=3.779 \mathrm{~V}$. As the reference electrode is chosen as an electron solvated in electrolyte far away from graphene in JDFT [33], the zero charge potential can be taken as a reference to discuss the negatively and positively charged states of the graphene for calculations with the same electrolyte. For example, Figure 2B illustrates the DOS of the negatively charged graphene with the excess charge of $-0.15|e|$ for the representative unit, $\bar{\mu}^{(W)}=-0.804 \mathrm{eV}$ and $\mathcal{E}-\mathcal{E}_{\mathrm{PZC}}=-2.975 \mathrm{~V}$, while Figure $2 \mathrm{C}$ indicates the DOS of the positively charged graphene with the excess charge of $0.22|e|$ for the representative unit, $\bar{\mu}^{(W)}=-7.495$ $e \mathrm{~V}$ and $\mathcal{E}-\mathcal{E}_{\mathrm{PZC}}=3.716 \mathrm{~V}$. The JDFT calculation results exhibit obviously the shift of the Dirac point with excess charge, which is consistent with experiments [5, 10, 42]. Negative (or positive) charge makes the Dirac point shift up (or down), as shown in Figure $2 \mathrm{~B}$ and $2 \mathrm{C}$. Although negative (or positive) charge makes the energy level of the Dirac point positive (or negative) compared with that of the charge-free state, the electrode potential becomes negative (or positive) with respect to the zero charge potential, as schematically shown in Figure 1C and 1D. The magnitude of the shift increases 
monotonically with the absolute value of excess charge, as shown in Figure 3 for two series of calculations with different nominal dielectric constant and ion concentration.

According to the quantum capacitance theory [43], the shift of the Dirac point is attributed to an electrostatic potential $\phi$ induced by the double layer distributions of charges/ions at the graphene/electrolyte interface and $-|e| \phi=\Delta \varepsilon$ [44]. This hypothesis has been employed in the theoretical study [26]. However, it has not been experimentally and/or numerically verified yet. To distinct the physical picture, onedimensional (1D) electrostatic potential along the $z$ direction was computed by planeaveraging of $3 \mathrm{D}$ electrostatic potential distribution, $\phi(z)=\sum_{i}^{N} \phi_{z i} / N(z)$, where $N(z)$ is the number of discrete points on a plane perpendicular to the $z$ direction and $\phi_{z i}$ is the potential value at point $i$. Furthermore, the relative 1D electrostatic potential distribution is computed by $\Delta \phi=\phi(z)-\phi_{0}(z)$, where $\phi(z)$ and $\phi_{0}(z)$ denote the potential distributions in the computed systems with charged graphene and charge-free graphene, respectively. Figure 4A shows four such relative potential distributions with four values of excess charges in the representative graphene, indicating that negative (or positive) charges produce a negative (or positive) electric field. The high potential region is within the graphene with a big dropping from the middle to the interface and inside the electrolyte the potential approaches zero in regions far away from the graphene. The negative (or positive) charges in graphene will induce positive (or negative) bound charges on the graphene surfaces, which forms one side of the double layers. The attracted positive (or negative) bound charges screen the negative (or positive) charges and make the big dropping in the electrostatic potential. Nevertheless, the negative (or positive) electrostatic potential must raise the energy of electrons in graphene and makes the Dirac point shifts upwards (or downwards), as shown in Figure $2 \mathrm{~B}$ (or 2C). The averaged potential $\overline{\Delta \phi}=\frac{\int_{-z_{1}}^{z_{1}} \Delta \phi(z) d z}{\int_{-z_{1}}^{z_{1}} d z}$ has the property that $|e| \overline{\Delta \phi}$ is linearly proportional to the shift of the Dirac point, $\Delta \varepsilon$, for the all computed cases, including four ionic concentrations and six values of the nominal dielectric constant of the electrolyte, as shown in Figure 4B for three calculated cases. However, the slope of $\Delta \varepsilon$ versus $-|e| \overline{\Delta \phi}$ depends on the value of $z_{1}$. Thus, the thickness of graphene might be defined based on $-|e| \overline{\Delta \phi}=\Delta \varepsilon$, which gives $z_{1} \approx 1.482 \AA$, as illustrated in Figure $4 \mathrm{~A}$. 
Summing up all bound charges in a plane parallelling graphene plane gives the plane-averaged bound charge distribution along the $z$ direction. Figure 5A indicates the original bound charge distributions for graphene without any excess charges and with four values of excess charges, $-0.15|e|,-0.06|e|, 0.10|e|$, and $0.22|e|$ in the representative graphene. The original bound charge distributions reveal the double layer configuration. Especially, the positive charge distribution is completely symmetric to the negative charge distribution for the graphene without any excess charges, implying the electrochemical nature of graphene in the electrolyte. When the graphene is negatively (or positively) charged, the positive (or negative) bound charge peak will increase and meanwhile the negative (or positive) peak will decrease. The change magnitude in the bound charge distribution monotonically increases with the amount of excess charges. Since the charge-free state is taken as reference in the electrochemical analysis, the relative bound charge distributions, which are the corresponding original bound charge distributions minus the original bound charge distribution for the chargefree graphene, are plotted in Figure 5B, exhibiting only one peak for each such curve. Combining Figure 5B with Figure 4A indicates that the electrons (or holes) and the bound charges form the double layers and the bound charges possess the same amount charges, but with an opposite sign as the electrons (or holes).

Figure 6A shows the computed excess charge versus the electrode potential with respect to the charge-free potential for four used electrolytes and the excess charge versus the potential induced by the chemical potential of electrons, which takes the Dirac point of charge-neutral graphene as reference. The previous theoretical study presented qualitatively the same tendency of the charge-potential relationship along with the change in ion concentration [26]. As indicated in Figure 2A-C, the shape of graphene DOS is like two circular cones linked at the two cone tips of the Dirac point. Thus, near the Dirac point, the curve of charge $q$ versus $-\mu /|e|$ can be expressed by $q=\alpha \cdot \operatorname{sign}(-\mu /|e|) \cdot(-\mu /|e|)^{2}$ with $\alpha$ being a constant independent of the electrolyte, as shown in Figure 6A. This equation can be alternately written as $-\mu /|e|=c \sqrt{q}$ for $q>0$ and $-\mu /|e|=-c \sqrt{-q}$ for $q<0$ with $c=\sqrt{\alpha}$ and a similar expression can be found in the literature [45]. Under an applied electrode potential, however, the Dirac point shifts and the excess charge varies also, which makes the 
relationship between excess charge and the relative electrode potential complicated. For simplification, an approximate expression of the Dirac point shift with the excess charge, $\Delta \varepsilon /|e|=a q+b q^{2}$, was derived in Appendix with $a$ and $b$ being electrolytedependent parameters. Then, the excess charge versus the relative electrode potential is approximately expressed as

$$
\mathcal{E}-\mathcal{E}_{\mathrm{PZC}}=-\frac{\mu+\Delta \varepsilon}{|e|}= \pm c \sqrt{ \pm q}-\left(a q+b q^{2}\right)
$$

while $c$ is intrinsic to graphene, $a$ and $b$ are determined by the electrolyte. The first term in the formula takes $-c \sqrt{-q}$ when $q<0$ and $c \sqrt{q}$ when $q>0$. The JDFT results in Figure 6A indicate that the higher the ion concentration and the higher the nominal dielectric constant is, the more the excess charges will be. This is because an electrolyte with a higher nominal dielectric constant and a higher ion concentration produces a higher compensating electrostatic potential to the potential produced by the excess charges in the graphene, leading to a lower $|\Delta \phi|$, a lower $|\Delta \varepsilon|$ and a larger $|\mu|$. As a direct consequence, the least number of excess charges can be introduced into graphene when it is in vacuum. This is because the Dirac point shift is only related to $\phi$ and is the largest in vacuum under a given applied electrode potential in comparison with other electrolytes.

The total capacitance of the electrochemical cell and the integral quantum and double layer capacitances are computed by

$$
C_{\text {total }}^{J D F T}=\frac{q / S}{\mathcal{E}-\mathcal{E}_{\mathrm{PZC}}}, \quad C_{d l}^{J D F T}=\frac{q / S}{-\Delta \varepsilon /|e|}, \quad C_{Q}^{J D F T}=\frac{q / S}{-\mu /|e|}
$$

respectively, where $S$ is the area of graphene in the representative cell and the superscript "JDFT" indicates that the capacitances are computed based on the JDFT results. With the approximate expression of the Dirac point shift with respect to the excess charge, the three capacitances are explicitly and analytically expressed by

$$
\begin{aligned}
C_{\text {total }}^{A} & =\frac{q / S}{ \pm c \sqrt{ \pm q}-\left(a q+b q^{2}\right)} \\
C_{d l}^{A} & =\frac{-q}{S\left(a q+b q^{2}\right)} \\
C_{Q}^{A} & =\frac{\sqrt{ \pm q}}{ \pm c S}=\frac{1}{\alpha S}(-\mu /|e|)
\end{aligned}
$$


where the superscript " $A$ " indicates that the capacitances are analytically determined with the proposed formulas.

It should be pointed out that the capacitance $C_{d l}$ is not a constant for a given electrochemical cell, because it depends on the charge, which in turn depends on the applied electrode potential, as also suggested in the theorectical work [26]. This result is a nature extension of the classical capacitance in an electrochemical cell including the Gouy-Chapman capacitance and the capacitance of the Stern layer, which is different from the case that graphene is an electrode in a solid capacitor [17, 19, 22].

Figures 6B-D show the computed $C_{\text {total }}^{J D F T}, C_{d l}^{J D F T}$ and $C_{Q}^{J D F T}$, respectively. Equation (5) indicates that $C_{Q}$ is indeed linearly proportional to $-\mu /|e|$, which is also plotted in Figure 6D. From the linear relationship and the computed results, we determined $c=4.2563 \mathrm{eV} / \sqrt{|e|}$. The differential quantum capacitance gives also the linear relationship between $C_{Q}$ and $\mu$ [40], with which and the JDFT results, we determined the value of Fermi velocity $v_{F}=0.8 \times 10^{6} \mathrm{~m} / \mathrm{s}$, in good agreement with the previous results [31]. The change rate of $C_{\text {total }}$ vs $\mathcal{E}-\mathcal{E}_{\mathrm{PZC}}$ is about $8.0 \mu \mathrm{F} /\left(\mathrm{cm}^{2} \cdot \mathrm{V}\right)$ within the potential range of $\pm 1 \mathrm{~V}$ in our computations with $0.05 \mathrm{M}$ ion concentration. This value is commensurate with the experimentally measured values of about 7.3 and 5.7 $\mu \mathrm{F} /\left(\mathrm{cm}^{2} \cdot \mathrm{V}\right)$ for graphene in $1 \mathrm{mM}$ and $0.5 \mathrm{mM} \mathrm{NaF}$ solutions, respectively, because high ion concentration generally leads to a large change rate [19]. We have calculated the values of $a$ and $b$ for various electrolytes and tabulated them in Table 1. Figure 3 also shows two examples of the fitted curves of $a$ and $b$. Using the determined values of $a$ and $b$ and undeformed C-C bond length of $1.420 \AA$, we predict the capacitances $C_{t o t a l}$ and $C_{d l}$ with Equation (1), (3) and (4). The predicted curves are plotted in Figure $6 \mathrm{~B}$ and $6 \mathrm{C}$, respectively. The predicted curves of the charge $q$ versus the relative potential $\mathcal{E}-\mathcal{E}_{\mathrm{PZC}}$ were also plotted in Figure A1 in Appendix. Obviously, the analytic expressions of the capacitances agree perfectly with the computed results.

The present work and the previous theoretical work [26] take almost the same approach to dissect capacitances at the graphene/electrolyte interface by combining DOS shape of graphene and modified Poisson-Boltzmann equation. Benefiting from the DFT calculations of graphene electrode, the present work is able to present the number of excess charges, Dirac point shift and electrostatic potential distribution simultane- 
ously, which makes it possible to examine the quantum capacitance theory in detail. The advantage of the theoretical work [26] lies in its analysis. Dirac point shift $\Delta \varepsilon$ is directly assigned to be the surface potential, based on which the chemical potential of electrons $\mu$ was calculated and then the number of excess charges was evaluated [26].

\section{Concluding remarks}

The proposed analytic formulas of capacitances are simple and involve only three parameters. The two parameters of $a$ and $b$ are electrolyte-dependent and parameter $c$ presents an intrinsic property of graphene, which reflects how much charge the graphene can accommodate when the Fermi level is deviated from the Dirac point. In addition, the joint first-principles/continuum calculations exhibit a quadratic relationship between Dirac point shift and excess charges in graphene under an applied voltage across the graphene and the reference electrode. The Fermi level change, Dirac point shift, electrostatic potential distribution and excess charges on graphene are all coupled one to another. The values of electrolyte-dependent parameters $a$ and $b$ are determined from the calculations for various electrolytes. The directly computed capacitances verify the proposed formulas. Further work is in progress to theoretically and analytically investigate the parameters $a$ and $b$.

\section{Acknowledgments}

The work was supported by the Hong Kong Research Grants Council through NSFC/RGC Joint Research Scheme with project N_HKUST621/11. S. Sun also acknowledges support by the National Natural Science Foundation of China (Grant No. 11202067) and the Fundamental Research Funds for the Central Universities (No. 2102B03214). Qi acknowledges support for analyzing data as part of the Nanostructures for Electrical Energy Storage (NEES), an Energy Frontier Research Center funded by the U.S. Department of Energy, Office of Science, Basic Energy Sciences under Award number DESC0001160. 


\section{Appendix. Supplementary Material}

Supplementary material associated with this article can be found online, including (1) Basic equations of JDFT, (2) Derivation of the approximate relationship between excess charges and Dirac point shift, and (3) Theoretical prediction and calculated data of the charge $q$ versus the relative potential $\mathcal{E}-\mathcal{E}_{\mathrm{PZC}}$.

\section{References}

[1] J. Slonczewski, P. Weiss, Band Structure of Graphite, Phys. Rev. 109 (2) (1958) 272-279. doi:10.1103/PhysRev.109.272. URL http://link.aps.org/doi/10.1103/PhysRev.109.272

[2] P. Wallace, The Band Theory of Graphite, Phys. Rev. 71 (9) (1947) 622-634. doi:10.1103/PhysRev.71.622. URL http://link.aps.org/doi/10.1103/PhysRev.71.622

[3] M. F. El-Kady, V. Strong, S. Dubin, R. B. Kaner, Laser scribing of highperformance and flexible graphene-based electrochemical capacitors., Science 335 (6074) (2012) 1326-1330. doi:10.1126/science.1216744.

URL http://www.ncbi.nlm.nih.gov/pubmed/22422977

[4] F. Chen, Q. Qing, J. Xia, J. Li, N. Tao, Electrochemical gate-controlled charge transport in graphene in ionic liquid and aqueous solution., J. Am. Chem. Soc. 131 (29) (2009) 9908-9909. doi:10.1021/ja9041862. URL http://www.ncbi.nlm.nih.gov/pubmed/19572712

[5] J. Ye, M. F. Craciun, M. Koshino, S. Russo, S. Inoue, H. Yuan, H. Shimotani, A. F. Morpurgo, Y. Iwasa, Accessing the transport properties of graphene and its multilayers at high carrier density. Proc. Natl. Acad. Sci. U. S. A. 108 (32) (2011) 13002-13006. doi:10.1073/pnas.1018388108.

URL http://www.pubmedcentral.nih.gov/articlerender. fcgi?artid=3156196\&tool=pmcentrez\&rendertype=abstract 
[6] R. Guidelli, W. Schmickler, Recent developments in models for the interface between a metal and an aqueous solution, Electrochim. Acta 45 (15-16) (2000) 2317-2338. doi:10.1016/S0013-4686(00)00335-2

URL http://linkinghub.elsevier.com/retrieve/pii/ S0013468600003352

[7] N. Luque, W. Schmickler, The electric double layer on graphite, Electrochim. Acta 71 (2012) 82-85. doi:10.1016/j.electacta.2012.03.083

URL http://linkinghub.elsevier.com/retrieve/pii/ S0013468612004355

[8] A. K. Geim, Graphene: status and prospects. Science 324 (5934) (2009) 15301534. doi:10.1126/science.1158877.

URL http://www.ncbi.nlm.nih.gov/pubmed/19541989

[9] G. Jo, M. Choe, S. Lee, W. Park, Y. H. Kahng, T. Lee, The application of graphene as electrodes in electrical and optical devices. Nanotechnology 23 (11) (2012) 112001. doi:10.1088/0957-4484/23/11/112001. URL http://www.ncbi.nlm.nih.gov/pubmed/22370228

[10] P. Wei, N. Liu, H. R. Lee, E. Adijanto, L. Ci, B. D. Naab, J. Q. Zhong, J. Park, W. Chen, Y. Cui, Z. Bao, Tuning the Dirac point in CVD-grown graphene through solution processed n-type doping with 2-(2-methoxyphenyl)-1,3dimethyl-2,3-dihydro-1H-benzoimidazole., Nano Lett. 13 (5) (2013) 1890-1897. doi:10.1021/n1303410g.

URL http://pubs.acs.org/doi/full/10.1021/ nl3034loghttp://www.ncbi.nlm.nih.gov/pubmed/23537351

[11] I. Gierz, C. Riedl, U. Starke, C. R. Ast, K. Kern, Atomic hole doping of graphene., Nano Lett. 8 (12) (2008) 4603-4607. doi:10.1021/n1802996s. URL http://www.ncbi.nlm.nih.gov/pubmed/19053796

[12] Y. Y. Wang, T. D. Pham, K. Zand, J. Li, P. J. Burke, Charging the quantum capacitance of graphene with a single biological ion channel., ACS Nano 8 (5) (2014) 
4228-4238. doi:10.1021/nn501376z.

URL http://www.ncbi.nlm.nih.gov/pubmed/24754625

[13] B. Mailly-Giacchetti, A. Hsu, H. Wang, V. Vinciguerra, F. Pappalardo, L. Occhipinti, E. Guidetti, S. Coffa, J. Kong, T. Palacios, pH sensing properties of graphene solution-gated field-effect transistors, J. Appl. Phys. 114 (8) (2013) 084505. doi:10.1063/1.4819219.

URL http://scitation.aip.org/content/aip/journal/jap/ $114 / 8 / 10.1063 / 1.4819219$

[14] P. K. Ang, W. Chen, A. T. S. Wee, K. P. Loh, Solution-gated epitaxial graphene as pH sensor., J. Am. Chem. Soc. 130 (44) (2008) 14392-14393. doi:10.1021/ ja805090z. URL http://www.ncbi.nlm.nih.gov/pubmed/18850701

[15] K. Xu, C. Zeng, Q. Zhang, R. Yan, P. Ye, K. Wang, A. C. Seabaugh, H. G. Xing, J. S. Suehle, C. A. Richter, D. J. Gundlach, N. V. Nguyen, Direct measurement of Dirac point energy at the graphene/oxide interface., Nano Lett. 13 (1) (2013) 131-136. doi:10.1021/n1303669w URL http://www.ncbi.nlm.nih.gov/pubmed/23244683

[16] S. Dröscher, P. Roulleau, F. Molitor, P. Studerus, C. Stampfer, K. Ensslin, T. Ihn, Quantum capacitance and density of states of graphene, Appl. Phys. Lett. 96 (15) (2010) 152104. doi:10.1063/1.3391670.

URL http://scitation.aip.org/content/aip/journal/apl/ 96/15/10.1063/1.3391670

[17] H. Xu, Z. Zhang, L.-M. Peng, Measurements and microscopic model of quantum capacitance in graphene, Appl. Phys. Lett. 98 (13) (2011) 133122. doi:10.1063/1.3574011.

URL http://scitation.aip.org/content/aip/journal/apl/ 98/13/10.1063/1.3574011

[18] F. Giannazzo, S. Sonde, V. Rainerl, E. Rimini, Screening length and quantum 
capacitance in graphene by scanning probe microscopy, Nano Lett. 9 (2009) 2329. doi:10.1021/n1801823n.

[19] J. Xia, F. Chen, J. Li, N. Tao, Measurement of the quantum capacitance of graphene., Nat. Nanotechnol. 4 (8) (2009) 505-509. doi:10.1038/nnano. 2009.177

URL http://www.ncbi.nlm.nih.gov/pubmed/19662012

[20] E. Uesugi, H. Goto, R. Eguchi, A. Fujiwara, Y. Kubozono, Electric double-layer capacitance between an ionic liquid and few-layer graphene. Sci. Rep. 3 (2013) 1595. doi:10.1038/srep01595.

URL http://www.pubmedcentral.nih.gov/articlerender. fcgi ?artid=3615339\&tool=pmcentrez\&rendertype=abstract

[21] L. A. Ponomarenko, R. Yang, R. V. Gorbachev, P. Blake, A. S. Mayorov, K. S. Novoselov, M. I. Katsnelson, A. K. Geim, Density of States and Zero Landau Level Probed through Capacitance of Graphene, Phys. Rev. Lett. 105 (13) (2010) 136801. doi:10.1103/PhysRevLett.105.136801.

URL http://link.aps.org/doi/10.1103/PhysRevLett.105. 136801

[22] G. L. Yu, R. Jalil, B. Belle, A. S. Mayorov, P. Blake, F. Schedin, S. V. Morozov, L. A. Ponomarenko, F. Chiappini, S. Wiedmann, U. Zeitler, M. I. Katsnelson, A. K. Geim, K. S. Novoselov, D. C. Elias, Interaction phenomena in graphene seen through quantum capacitance., Proc. Natl. Acad. Sci. U. S. A. 110 (9) (2013) 3282-3286. doi:10.1073/pnas.1300599110. URL http://www.ncbi.nlm.nih.gov/pubmed/23401538

[23] S. Dröscher, P. Roulleau, F. Molitor, P. Studerus, C. Stampfer, K. Ensslin, T. Ihn, Quantum capacitance and density of states of graphene. Phys. Scr. T146 (2012) 014009. doi:10.1088/0031-8949/2012/T146/014009.

URL http://stacks.iop.org/1402-4896/2012/i=T146/a= 014009 ?key=crossref.c524efed8f64cf675c25390150700604 
[24] A. A. Kornyshev, Double-layer in ionic liquids: paradigm change?, J. Phys. Chem. B 111 (20) (2007) 5545-5557. doi:10.1021/ jp0678570

URL/http://www.ncbi.nlm.nih.gov/pubmed/17469864

[25] K. B. Oldham, A Gouy-Chapman-Stern model of the double layer at a (metal)/(ionic liquid) interface, J. Electroanal. Chem. 613 (2) (2008) 131-138. doi:10.1016/j.jelechem.2007.10.017.

URL http://linkinghub.elsevier.com/retrieve/pii/ S002207280700486X

[26] Z. L. Mišković, N. Upadhyaya, Modeling Electrolytically Top-Gated Graphene. Nanoscale Res. Lett. 5 (3) (2010) 505-511. doi: $10.1007 / \mathrm{s} 11671-009-9515-3$.

URL http://ww.pubmedcentral.nih.gov/articlerender. fcgi?artid=2894001\&tool=pmcentrez\&rendertype=abstract

[27] M. Topsakal, S. Ciraci, Static charging of graphene and graphite slabs, Appl. Phys. Lett. 98 (13) (2011) 131908. doi:10.1063/1.3573806.

URL http://link.aip.org/link/APPLAB/v98/i13/p131908/ s1\&Agg=doi

[28] M. Topsakal, S. Ciraci, Effects of static charging and exfoliation of layered crystals Phys. Rev. B 85 (4) (2012) 045121. doi:10.1103/PhysRevB.85. 045121.

URL http://link.aps.org/doi/10.1103/PhysRevB.85.045121

[29] M. Topsakal, H. H. Gürel, S. Ciraci, Effects of Charging and Electric Field on Graphene Oxide, J. Phys. Chem. C 117 (11) (2013) 5943-5952. doi: $10.1021 / j p 310352 u$.

URL http://pubs.acs.org/doi/abs/10.1021/jp310352u

[30] M. Otani, O. Sugino, First-principles calculations of charged surfaces and interfaces: A plane-wave nonrepeated slab approach. Phys. Rev. B 73 (11) (2006) 115407. doi:10.1103/PhysRevB.73.115407.

URL http://link.aps.org/doi/10.1103/PhysRevB.73.115407 
[31] B. C. Wood, T. Ogitsu, M. Otani, J. Biener, First-Principles-Inspired Design Strategies for Graphene-Based Supercapacitor Electrodes, J. Phys. Chem. C 118 (1) (2014) 4-15. doi:10.1021/jp4044013

URL http://pubs.acs.org/doi/abs/10.1021/jp4044013

[32] S. A. Petrosyan, A. A. Rigos, T. A. Arias, Joint density-functional theory: ab initio study of $\mathrm{Cr}_{2} \mathrm{O}_{3}$ surface chemistry in solution. J. Phys. Chem. B 109 (32) (2005) 15436-15444. doi:10.1021/jp044822k

URL http://www.ncbi.nlm.nih.gov/pubmed/16852958

[33] K. Letchworth-Weaver, T. A. Arias, Joint density functional theory of the electrode-electrolyte interface: Application to fixed electrode potentials, interfacial capacitances, and potentials of zero charge, Phys. Rev. B 86 (7) (2012) 075140. arXiv:1205.0526, doi:10.1103/PhysRevB.86.075140 URL http://arxiv.org/abs/1205.0526http://link.aps.org/ doi/10.1103/PhysRevB.86.075140

[34] R. Sundararaman, K. Letchworth-Weaver, T. A. Arias, JDFTx, available at http://jdftx.sourceforge.net (2012).

URL http://jdftx.sourceforge.net

[35] J. Perdew, A. Ruzsinszky, G. Csonka, L. Constantin, J. Sun, Workhorse Semilocal Density Functional for Condensed Matter Physics and Quantum Chemistry, Phys. Rev. Lett. 103 (2) (2009) 026403. doi: $10.1103 /$ PhysRevLett.103.026403.

URL http://link.aps.org/doi/10.1103/PhysRevLett.103. 026403

[36] J. Sun, M. Marsman, A. Ruzsinszky, G. Kresse, J. P. Perdew, Improved lattice constants, surface energies, and $\mathrm{CO}$ desorption energies from a semilocal density functional Phys. Rev. B 83 (12) (2011) 121410. doi:10.1103/PhysRevB. 83.121410 .

URL http://link.aps.org/doi/10.1103/PhysRevB.83.121410 
[37] N. Troullier, J. L. Martins, Efficient pseudopotentials for plane-wave calculations, Phys. Rev. B 43 (3) (1991) 1993-2006. doi:10.1103/PhysRevB. 43.1993.

URL http://link.aps.org/doi/10.1103/PhysRevB.43.1993

[38] H. J. Monkhorst, J. D. Pack, Special points for Brillouin-zone integrations, Phys. Rev. B 13 (12) (1976) 5188-5192. doi:10.1103/PhysRevB.13.5188. URL http://link.aps.org/doi/10.1103/PhysRevB.13.5188

[39] C. Freysoldt, S. Boeck, J. Neugebauer, Direct minimization technique for metals in density functional theory, Phys. Rev. B 79 (24) (2009) 241103. doi:10. 1103/PhysRevB.79.241103. URL http://link.aps.org/doi/10.1103/PhysRevB.79.241103

[40] A. H. Castro Neto, N. M. R. Peres, K. S. Novoselov, A. K. Geim, The electronic properties of graphene, Rev. Mod. Phys. 81 (1) (2009) 109-162. doi: 10.1103/RevModPhys.81.109. URL http://link.aps.org/doi/10.1103/RevModPhys.81.109

[41] G. Lehmann, M. Taut, On the Numerical Calculation of the Density of States and Related Properties. Phys. Status Solidi 54 (2) (1972) 469-477. doi:10.1002/ pssb.2220540211.

URL http://doi.wiley.com/10.1002/pssb.2220540211

[42] K. Pi, K. McCreary, W. Bao, W. Han, Y. Chiang, Y. Li, S.-W. Tsai, C. Lau, R. Kawakami, Electronic doping and scattering by transition metals on graphene, Phys. Rev. B 80 (7) (2009) 075406. doi : 10 . 1103 / PhysRevB. 80 . 075406. URL http://link.aps.org/doi/10.1103/PhysRevB.80.075406

[43] S. Luryi, Quantum capacitance devices, Appl. Phys. Lett. 52 (6) (1988) 501. doi:10.1063/1.99649,

URL http://link.aip.org/link/APPLAB/v52/i6/p501/s1\& Agg $=$ doi 
[44] J. Fernández-Rossier, J. Palacios, L. Brey, Electronic structure of gated graphene and graphene ribbons, Phys. Rev. B 75 (20) (2007) 205441. arXiv: 0702473 , doi:10.1103/PhysRevB.75.205441.

URL http://arxiv.org/abs/cond-mat/0702473http://link. aps.org/doi/10.1103/PhysRevB.75.205441

[45] T. Fang, A. Konar, H. Xing, D. Jena, Carrier statistics and quantum capacitance of graphene sheets and ribbons. Appl. Phys. Lett. 91 (9) (2007) 092109. arXiv:0707.2242, doi:10.1063/1.2776887.

URL http://arxiv.org/abs/0707.2242http://scitation. aip.org/content/aip/journal/apl/91/9/10.1063/1. 2776887 
FIG. 1. (A) Schematic diagram of the electrochemical cell with graphene as the working electrode. A sustained applied potential $\mathcal{E}$ across the graphene electrode and the reference electrode can induce excess charges on graphene and meanwhile bound charge distributions in electrolyte. (B) The total capacitance of the electrochemical cell is composed of two in series capacitances, called the quantum capacitance $C_{Q}$ and the double layer capacitance $C_{d l}$. Accordingly, the applied potential is separated into two potentials, $-\mu /|e|$ acting on quantum capacitance $C_{Q}$ and $-\Delta \varepsilon /|e|$ acting on capacitance $C_{d l}$, where $\mu$ is the chemical potential of electrons in graphene referenced to the Dirac point, $\Delta \varepsilon$ denotes the value of the Dirac point shift, and $|e|$ stands for the absolute charge of an electron. (C) and (D) Relationships among variables in the circuit for $\mathcal{E}<\mathcal{E}_{\mathrm{PZC}}$ and $\mathcal{E}>\mathcal{E}_{\mathrm{PZC}}$, respectively, where $\bar{\mu}^{(W)}$ and $\bar{\mu}^{(R)}$ are the electron chemical potentials of working electrode and reference electrode, respectively; $\mathcal{E}_{\mathrm{PZC}}$ is the electrode potential when the graphene is in charge neutral state.

FIG. 2. The calculated densities of states of graphene with excess charges of (A) 0 , (B) $-0.15|e|$ and (C) $0.22|e|$, respectively, in the representative graphene monolayer immersed in the electrolyte possessing the nominal dielectric constant of $\epsilon=78.4$ and ion concentration of $1.0 \mathrm{M}$, where the dashed lines indicate the energy levels of Dirac point. Fermi level change and Dirac point shift are revealed.

FIG. 3. Dirac point shift vs excess charges in graphene electrode immersed in electrolyte with (A) the nominal dielectric constant of 78.4 and ion concentration of $1.0 \mathrm{M}$ and (B) the nominal dielectric constant of 3.1 and ion concentration of $0.5 \mathrm{M}$. The values are fitted by the formula $\Delta \varepsilon=a q+b q^{2}$.

FIG. 4. (A) Plane-averaged electrostatic potential distribution along the $z$ direction (perpendicular to the graphene plane) for four charged graphenes relative to the chargeneutral one. The electrolyte in these systems has the nominal dielectric constant of 78.4 and ion concentration of 1.0 M. (B) Dirac point shift vs thickness-averaged electrostatic potential for three systems. The relative potential averaged over the graphene thickness $\overline{\Delta \phi}=\frac{\int_{-z_{1}}^{z_{1}} \Delta \phi(z) d z}{\int_{-z_{1}}^{z_{1}} d z}$ has the property that $-|e| \overline{\Delta \phi}$ is linearly proportional to the shift of the Dirac point, $\Delta \varepsilon$, for all calculated cases. However, the slope of $\Delta \varepsilon$ vs $|e| \overline{\Delta \phi}$ depends on the value of $z_{1}$ and $-|e| \overline{\Delta \phi}=\Delta \varepsilon$ leads to $z_{1} \approx 1.482 \AA$. 
FIG. 5. (A) Double layer configurations of plane-averaged bound charge distribution without any excess charges and with four values of excess charges in the representative graphene. (B) Bound charge distributions in systems with charged graphene relative to that with charge neutral graphene, revealing a single peak distribution.

FIG. 6. (A) Excess charge vs the relative electrode potential, $\mathcal{E}-\mathcal{E}_{\mathrm{PZC}}$, with respect to the charge-free potential for the electrolyte possessing different nominal dielectric constants and ionic concentrations. The excess charge vs $-\mu /|e|$ is also plotted here for comparison. (B) Total capacitance, (C) double layer capacitance and (D) quantum capacitance in three calculated systems vs the relative electrode potential $\mathcal{E}-\mathcal{E}_{\mathrm{PzC}}$. Points are computed values by Equation (2) based on JDFT results. Solid curves in (B) and (C) are the predictions of $C_{t o t a l}$ and $C_{d l}$, respectively, from Equation (1), (3) and (4) with undeformed C-C bond length of $1.420 \AA$. It is also shown in (D) of quantum capacitance $C_{Q}$ as a function of $-\mu /|e|$ and solid curve is fitting result by using Equation (5). 
(A)

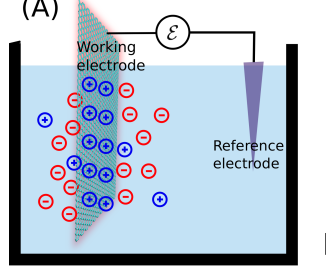

(B)

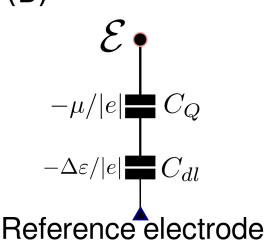

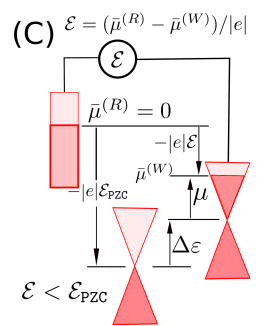

(D) $\mathcal{E}=\left(\bar{\mu}^{(R)}-\bar{\mu}^{(W)}\right) /|e|$

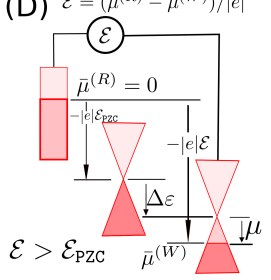

Figure 1:

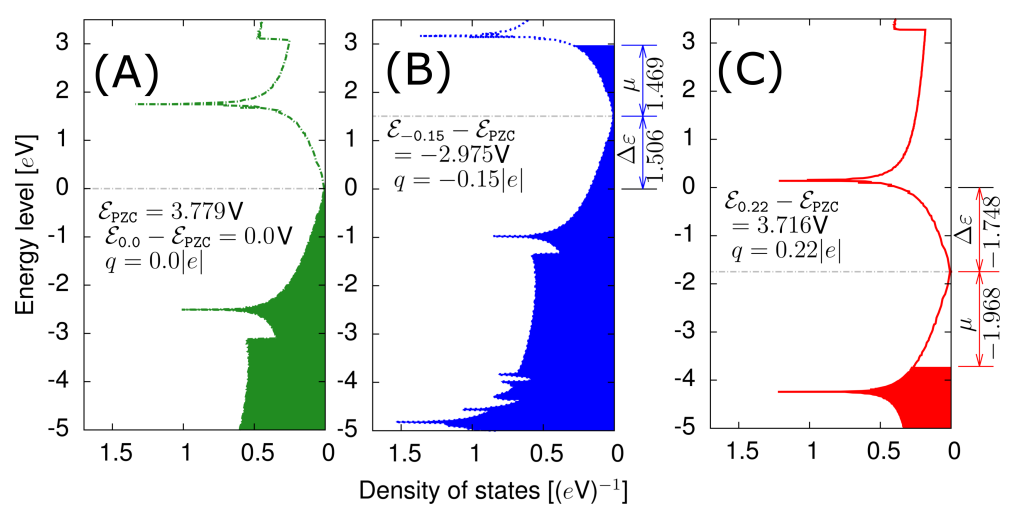

Figure 2:

Table 1: Values of $a$ and $b$ for various electrolytes.

\begin{tabular}{|c|c|c|c|}
\hline Dielectric constant $\epsilon$ & Ion concentration $(\mathrm{M})$ & $a(\mathrm{~V} / e)$ & $b\left(\mathrm{~V} / e^{2}\right)$ \\
\hline 78.4 & 0.05 & -12.0955 & 5.2662 \\
78.4 & 0.1 & -10.8535 & 5.36865 \\
78.4 & 0.5 & -9.5599 & 5.48069 \\
78.4 & 1.0 & -9.24459 & 5.73844 \\
64.0 & 0.5 & -9.88831 & 5.67459 \\
38.0 & 0.5 & -10.8404 & 6.14434 \\
7.6 & 0.5 & -14.7954 & 7.72014 \\
4.8069 & 0.5 & -16.2444 & 8.14078 \\
3.1 & 0.5 & -17.7613 & 8.47217 \\
\hline
\end{tabular}



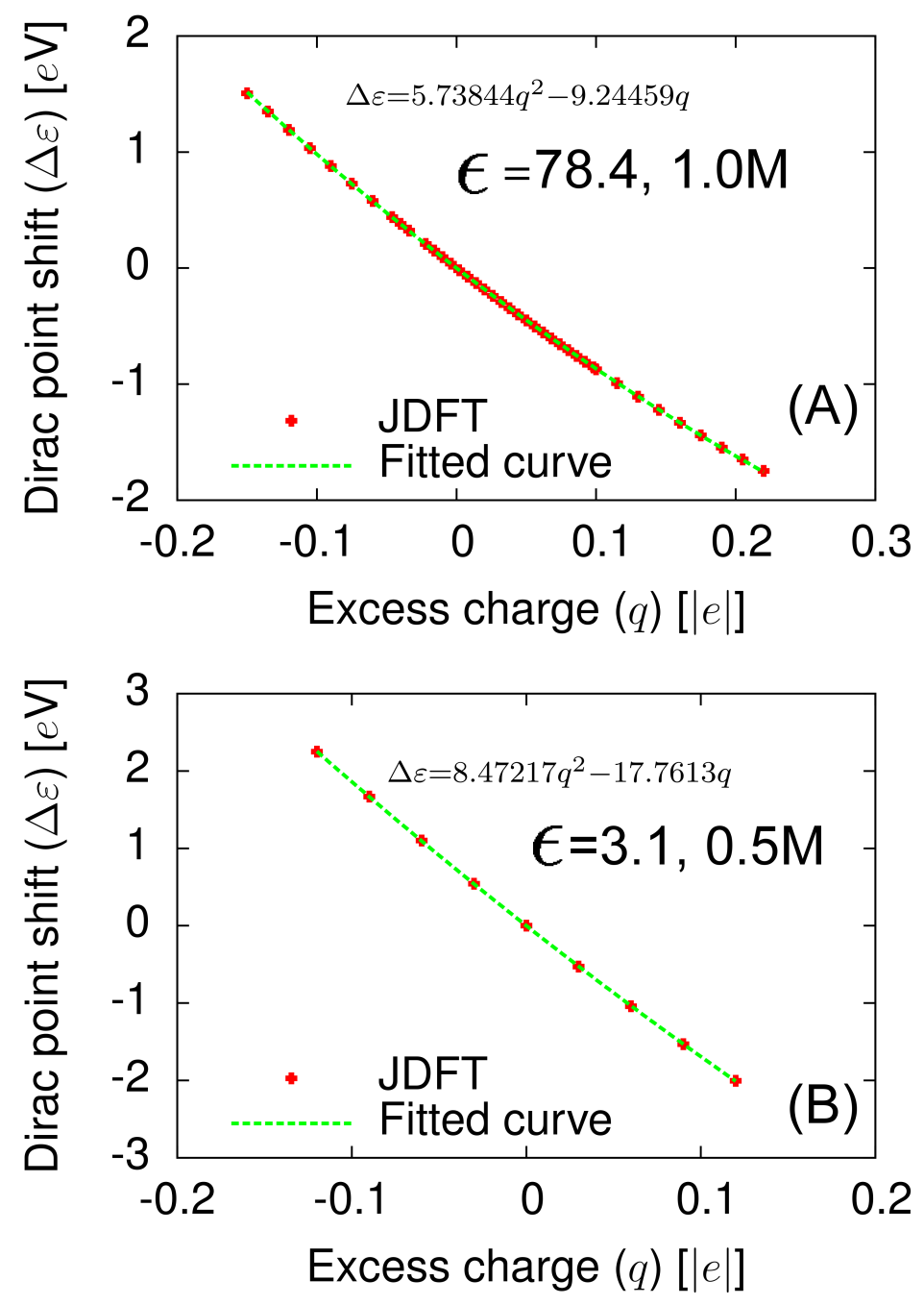

Figure 3: 

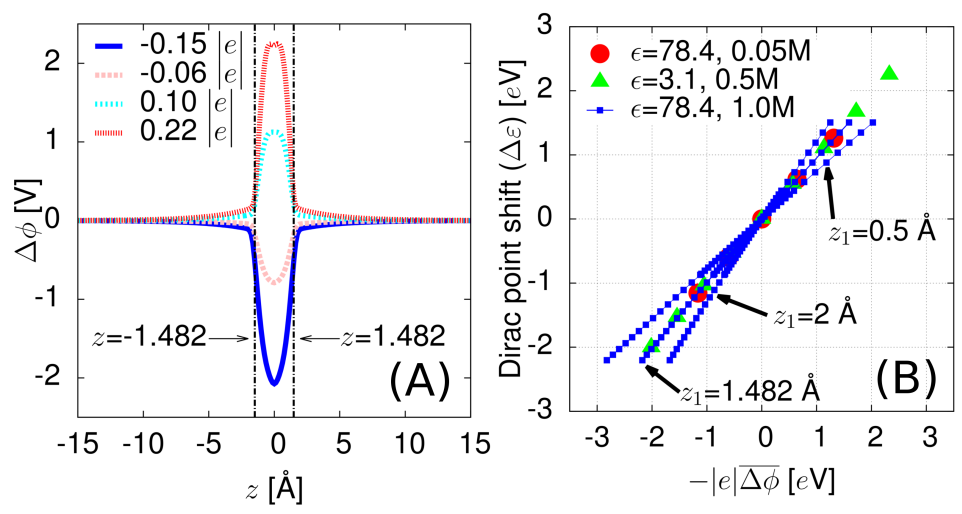

Figure 4: 

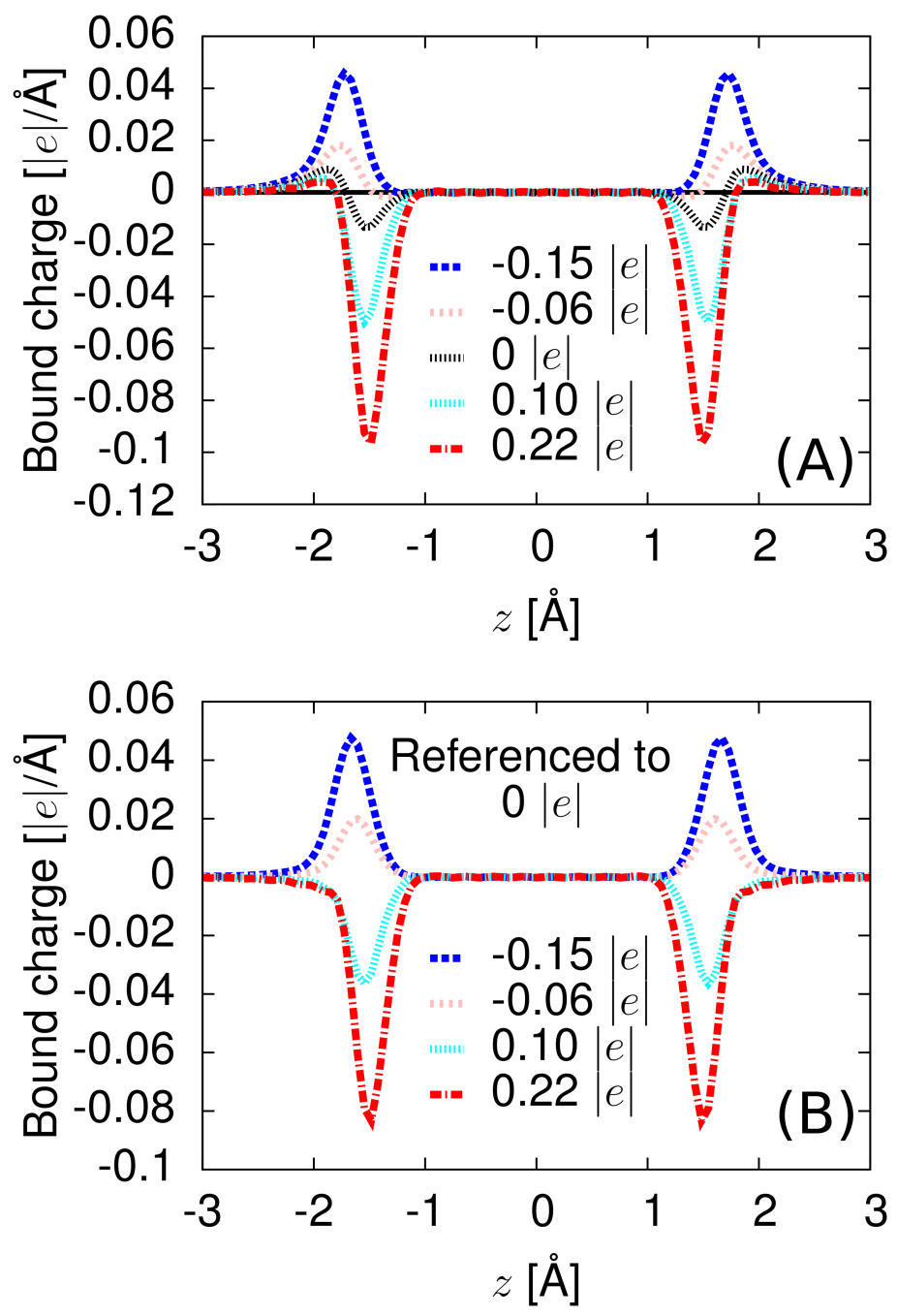

Figure 5: 

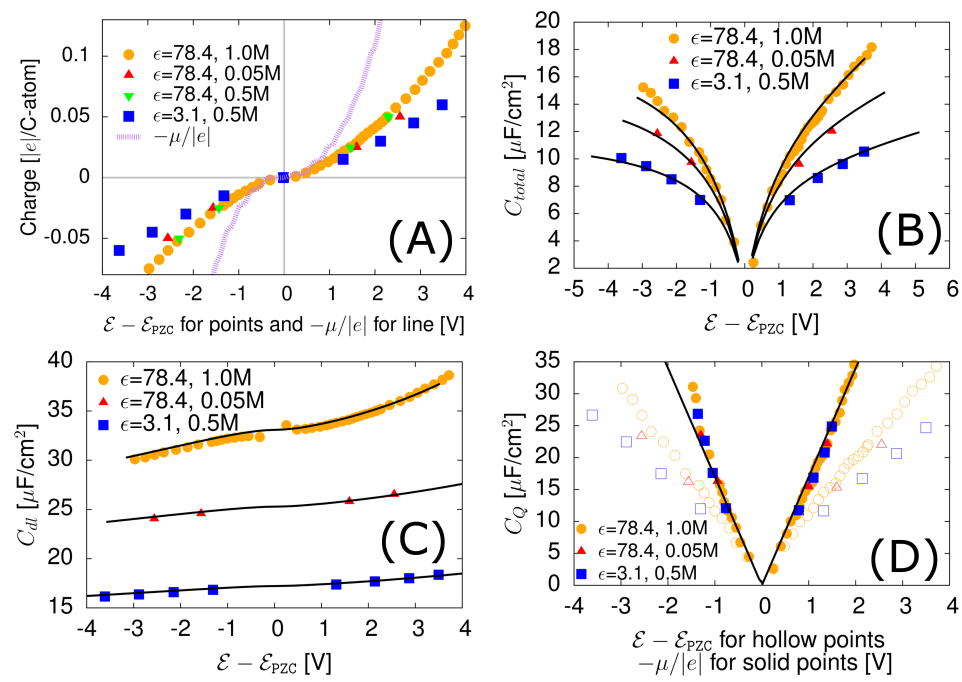

Figure 6: 